\title{
SÍ HAY NEGACIÓN LÓGICA
}

Ricardo Arturo Nicolás Francisco

Universidad Nacional Autónoma de México

Programa de Posgrado en Filosofía de la Ciencia gyl.ric@gmail.com

Luis Estrada GonZÁLEZ Universidad Nacional Autónoma de México Instituto de Investigaciones Filosóficas Programa de Posgrado en Filosofía de la Ciencia loisayaxsegrob@gmail.com

RESUMEN: En este artículo discutimos la tesis de Jc Beall según la cual no hay negación lógica. Evaluamos la solidez del argumento con el que defiende su tesis y presentamos dos razones para rechazar una de sus premisas: que la negación tiene que ser excluyente o exhaustiva. La primera razón involucra una presentación alternativa de las reglas de la negación en sistemas de secuentes diferentes al que Beall presupone. La segunda razón establece que la negación no tiene que ser excluyente o exhaustiva.

PALABRAS CLAVE: exclusividad, exhaustividad, FDE, separatismo, interaccionismo

SUMMARY: In this paper we discuss Jc Beall's thesis that there is no logical negation. We asses the soundness of Beall's argument and we put forward two reasons to negate the truth of one of its premises: that negation has to be whether exclusive or exhaustive. The first reason involves an alternative presentation of the rules for negation in sequent systems different to that presupposed by Beall. The second reason establishes that negation need not be neither exclusive nor exhaustive.

KEY WORDS: exclusivity, exhaustivity, FDE, separatism, interactionism

\section{Introducción}

En este artículo discutiremos la tesis de Beall, defendida en 2017, de que no hay negación lógica. Evaluaremos la solidez del argumento que sustenta la tesis y presentaremos dos razones para negar la verdad de una de sus premisas, según la cual la negación tiene que ser excluyente o exhaustiva. La primera razón que ofrecemos es que incluso si aceptamos que la negación en la lógica preferida por Beall - FDEtiene que estar caracterizada por reglas en las que no aparezcan otras conectivas, reglas así pueden obtenerse en marcos lógicos distintos (marcos de sistemas de secuentes tri y tetralaterales) al presupuesto 
por Beall, que es un marco de sistemas de secuentes de conclusiones múltiples. De hecho, hay reglas así para la negación en un cálculo de secuentes estándar que ni Beall ni sus detractores consideran. En ambos casos las reglas para la negación no expresan exclusividad ni exhaustividad. La segunda razón, todavía más sencilla, dice que la negación no tiene que caracterizarse mediante exclusividad o exhaustividad, sino simplemente como una conectiva que intercambia la verdad y la falsedad.

El problema que abordamos aquí es importante porque está estrechamente vinculado con la comprensión de FDE, que es una lógica central en muchas investigaciones lógicas contemporáneas, además de las del propio Beall. ${ }^{l}$ Sin embargo, la pertinencia del tema no se agota en la comprensión de FDE, sino que se trata de un problema más general acerca de la naturaleza de la negación. En ese sentido, nuestro trabajo puede enmarcarse en el debate reciente entre Omori $/ \mathrm{De}^{2}$ y Berto/Restall. ${ }^{3}$ Si bien no nos pronunciamos acerca de cuál es el mejor tratamiento semántico para la negación — si el "plan australiano" que Berto y Restall defienden con su semántica tipo Kripke y condiciones no estándar para la negación, o el "plan estadounidense" que Omori y De favorecen con una semántica extensional bivaluada pero no vertitativo funcional- sí tratamos el problema de qué propiedades sí cabe esperar en una teoría suficientemente general acerca de la negación.

La estructura del artículo es la siguiente. En la primera sección presentamos el argumento con el que Beall defiende que no hay negación lógica. En la segunda sección presentamos la objeción de Lionel Shapiro al argumento de Beall, de acuerdo con la cual al cambiar la presentación de la lógica a marcos de secuentes tri y tetralaterales se pueden encontrar reglas para la negación en las que no aparecen otras conectivas. En la tercera sección presentamos la segunda objeción, según la cual no es necesario que la negación tenga que ser excluyente o exhaustiva. La insistencia de Beall en que una conectiva lógica debe estar caracterizada por reglas en las que no aparezcan otras conectivas se asemeja a la postura separatista en el tema del significado de las conectivas lógicas. En la cuarta sección discutiremos este supuesto, lo cual también nos lleva a considerar que hay otras opciones antes de concluir la inexistencia de la negación lógica.

${ }^{1}$ Véanse, por ejemplo, Paoli 2019, Omori y Wansing 2017 y 2019.

${ }^{2} C f r$. De y Omori 2018.

${ }^{3}$ Cfr. Berto 2015 y Berto y Restall 2018. 


\section{El argumento sobre la inexistencia de la negación lógica}

Jc Beall (2017) ha defendido que no hay negación lógica. Su argumento para defender esta tesis es el siguiente:

B1. La negación lógica debe ser excluyente o exhaustiva, esto es, dadas un par de proposiciones $A$ y $\sim A$, no pueden tener el mismo valor (exclusividad) o entre las dos tienen todos los valores posibles (exhaustividad).

B2. Hay razones para abandonar exclusividad.

B3. Hay razones para abandonar exhaustividad.

B4. No hay razones para preferir abandonar exclusividad sobre exhaustividad, ni viceversa. Además, las razones para abandonar exclusividad y exhaustividad son más fuertes que las razones para aceptarlas.

B5. Si hay razones para abandonar exclusividad y hay razones para abandonar exhaustividad, no hay razones para preferir abandonar una sobre la otra, y las razones para abandonar exclusividad y exhaustividad son más fuertes que las razones para aceptarlas, entonces hay que abandonar ambas.

B6. Entonces, hay que abandonar exclusividad y exhaustividad.

B7. Así, la negación lógica no es excluyente ni exhaustiva.

BC. Por lo tanto, no hay negación lógica.

A continuación discutiremos brevemente todas las premisas, excepto la primera, que dejaremos para la sección 3.

Beall dice que las razones para abandonar exclusividad las proporcionan las oraciones paradójicas de autorreferencia. Una de estas oraciones es la del mentiroso: "Esta oración es falsa". Para interpretar esta oración en términos intuitivos, las personas que admiten cúmulos de valores de verdad (por ejemplo, Priest 2006, Beall 2009, Weber 2010, Weber y col. 2014), usan una semántica que les permite expresar que hay oraciones que son tanto verdaderas como falsas. Dado que ellos interpretan la negación de una proposición como la afirmación de su falsedad, abandonan el requerimiento de que la negación sea excluyente de la verdad de esa fórmula. La lógica preferida por varias personas que admiten cúmulos de valores de verdad para hacer esto es la lógica paraconsistente $\mathbf{L P}{ }^{4}{ }^{4}$

Con respecto a la tercera premisa, Beall dice que los fenómenos de vaguedad proporcionan las razones para abandonar exhaustividad.

${ }^{4}$ Las propiedades de $\mathbf{L P}$ han sido ampliamente estudiadas en varios lugares y son bien conocidas; el locus classicus es por supuesto Priest 1979; el lector con menos tiempo libre puede revisar Priest 2006, capítulo 5. 
Para hablar de los fenómenos de vaguedad, las personas que admiten vacíos de valores de verdad (por ejemplo, McGee 1991 o Field 2008) usan una semántica que les permita expresar que hay oraciones que no son ni verdaderas ni falsas. La lógica preferida por varias personas que admiten vacíos de valores de verdad para hacer esto es la lógica paracompleta $\mathbf{K}_{3} .^{5}$

Con respecto a la cuarta premisa, Beall dice que no hay razones dadas puramente por la lógica para preferir abandonar exclusividad sobre exhaustividad, ni viceversa. Las únicas razones para preferir abandonar exclusividad sobre exhaustividad (o viceversa) son razones extralógicas, como que haya oraciones paradójicas de autorreferencia o fenómenos de vaguedad. Sin embargo, tomadas en conjunto, éstas constituyen razones más fuertes para abandonar tanto exhaustividad como exclusividad, no solamente una de ellas. A Beall le parece que no hay una manera lógicamente imparcial para decidir entre exhaustividad y exclusividad sin que se acepte la posición que se quiere probar. Por ello es que Beall (2018) cree que la lógica que subyace a todas nuestras teorías verdaderas tiene que ser subclásica - esto es, una lógica cuyo conjunto de argumentos válidos sea un subconjunto del conjunto de argumentos válidos de la lógica clásicay dicha lógica tiene que tener una negación que no sea excluyente ni exhaustiva. Beall considera que esa lógica es FDE. ${ }^{6}$

Cualquiera de las premisas se puede discutir, pero le concederemos a Beall las premisas B2 a B7, de modo que nos concentraremos en la evaluación de Bl.

\section{Las reglas de derivación para la negación}

Beall toma como evidencia de que no hay negación lógica que las reglas para la negación en FDE sean las siguientes:

${ }^{5}$ Para un estudio sucinto de las características de $\mathbf{K}_{3}$, véase Field 2008, capítulo 3.

${ }^{6}$ Las lógicas $\mathbf{L P}$ y $\mathbf{K}_{3}$ son extensiones de $\mathbf{F D E}$. Hay varias maneras de obtener aquellas dos lógicas a partir de ésta; en la siguiente sección presentamos una forma de hacerlo. El argumento de Beall sugiere que si la teoría correcta acerca de la negación no es la de $\mathbf{K}_{3}$ ni la de $\mathbf{L P}$, quizá tal teoría correcta pueda obtenerse de la intersección de ambas lógicas. La intersección de $\mathbf{L P}$ y $\mathbf{K}_{3}$ es la lógica S3; véase Kremer 1988. S3 contiene estrictamente a FDE, pues en ella valen todos los argumentos válidos en FDE, pero también el siguiente, que es inválido en FDE:

(RA) $A \wedge \sim A \vdash B \vee \sim B$

No obstante, S3 no es la lógica favorecida por el argumento de Beall porque la validez de (RA) en esa lógica sugeriría que si la negación no es excluyente entonces es exhaustiva y, contraponiendo, que si no es exhaustiva entonces es excluyente, lo cual va contra la idea de que la negación no es excluyente ni exhaustiva. 


$$
\begin{array}{rlrl}
\sim \wedge \mathrm{L} \frac{\Gamma, \sim A \vdash \Delta \quad \Gamma, \sim B \vdash \Delta}{\Gamma, \sim(A \wedge B) \vdash \Delta} & \sim \wedge \mathrm{R} \frac{\Gamma \vdash \Delta, \sim A, \sim B}{\Gamma \vdash \Delta, \sim(A \wedge B)} \\
\sim \vee \mathrm{L} \frac{\Gamma, \sim A, \sim B \vdash \Delta}{\Gamma, \sim(A \vee B) \vdash \Delta} & \sim \vee \mathrm{R} \frac{\Gamma \vdash \Delta, \sim A \quad \Gamma \vdash \Delta, \sim B}{\Gamma \vdash \Delta, \sim(A \vee B)} \\
& \sim \sim \mathrm{L} \frac{\Gamma, A \vdash \Delta}{\Gamma, \sim \sim \vdash \Delta} & \sim \sim \mathrm{R} \frac{\Gamma \vdash \Delta, A}{\Gamma \vdash \Delta, \sim \sim A}
\end{array}
$$

Considerando reglas de este tipo, Beall concluye que "[...] no hay una negación lógica filosóficamente interesante (más allá de los patrones de De Morgan), ninguna conectiva de negación interesante cuyo comportamiento independiente [de otras conectivas] [...] esté caracterizado por la lógica misma" $(2017, \text { p. } 2)^{7}$ y "hay una conectiva lógica llamada negación u operador de falsedad, sólo que la lógica no le impone ninguna restricción interesante [...] aparte de lo que la lógica le demanda de su interacción con otras conectivas [que sí son] lógicas" (2017, p. 15). ${ }^{8} \mathrm{Al}$ considerar que en las reglas para una conectiva lógica — en este caso, la negación - no deben aparecer otras conectivas, Beall es implícitamente un separatista, pero trataremos este asunto en la sección 4.

Shapiro (2016a) ha replicado que Beall llegó a su conclusión por haber considerado sólo sistemas de secuentes con conclusiones múltiples. En cálculos de secuentes tri y tetralaterales (Shapiro 2016b) hay reglas para la negación incluso para la lógica FDE con los requisitos que Beall pide, esto es, que no involucren otras conectivas. Estos últimos sistemas son parecidos a los sistemas de secuentes de $n$ lados. $^{9}$

La motivación formal para desarrollar estos sistemas es la de proporcionar una contrapartida de secuentes para la teoría de modelos de lógicas con $n$ valores siguiendo esta intuición: si la lógica con dos valores necesita "secuentes bilaterales", $\Gamma \vdash \Delta$, una lógica con $n$ valores necesita de "secuentes $n$-laterales":

$$
\Gamma_{1}, A_{1_{1}}, \ldots, A_{1_{n}} ; \ldots ; \Gamma_{k}, A_{k_{1}}, \ldots, A_{k_{m}} \vdash \Delta_{1}, B_{1_{1}}, \ldots, B_{1_{n}} ; \ldots ; \Delta_{k}, B_{k_{1}}, \ldots, B_{k_{m}}
$$

${ }^{7}$ Todas las traducciones son nuestras.

${ }^{8}$ En otras palabras, para Beall una conectiva lógica debe ser caracterizada por medio de "reglas puras" — como se les llama en Marcos 2005- o más exactamente "reglas de introducción puras" en cálculos de secuentes, es decir, reglas en donde las conectivas sólo figuren en el secuente inferior. Estas nociones serán útiles cuando al final de la sección analicemos una última línea de defensa para alguien que piense como Beall.

${ }^{9}$ Véase Paoli 2002, capítulo 4. 
Así, en un marco de secuentes trilaterales, las reglas de derivación tienen una de las siguientes formas generales:

$$
\frac{\Gamma, A ; \Sigma, B \vdash \Delta, C}{\Theta, D ; \Phi, E \vdash \Psi, F} \quad \frac{\Gamma, A \vdash \Delta, B ; \Sigma, C}{\Theta, D \vdash \Psi, E ; \Phi, F}
$$

donde $\Gamma$ y $\Theta$ no pueden ser vacíos.

En el caso particular de las reglas para las conectivas en FDE usamos la forma de la izquierda; en el caso de la negación, tenemos las siguientes reglas de derivación:

$$
\sim \mathrm{R}^{\prime} \frac{\Gamma ; \Sigma, A \vdash \Delta}{\Gamma ; \Sigma \vdash \Delta, \sim A} \quad \sim \mathrm{L}^{\prime} \frac{\Gamma ; \Sigma \vdash \Delta, A}{\Gamma ; \Sigma, \sim A \vdash \Delta}
$$

Si permitiéramos que $A$ estuviera del lado de $\Gamma$ en la regla $\sim$ R', es decir,

$$
\sim \mathrm{R}^{*} \frac{\Gamma, A ; \Sigma \vdash \Delta}{\Gamma ; \Sigma \vdash \Delta, \sim A}
$$

obtendríamos la negación de LP. Si permitiéramos que $\sim A$ estuviera del lado de $\Gamma$ en la regla $\sim$ L', es decir,

$$
\sim \mathrm{L}^{*} \frac{\Gamma ; \Sigma \vdash \Delta, A}{\Gamma, \sim A ; \Sigma \vdash \Delta}
$$

obtendríamos la negación de $\mathbf{K}_{3}$. Si permitiéramos las cuatro reglas anteriores, obtendríamos la negación de la lógica clásica. En este caso no tendría sentido, más allá de la generalidad, usar el punto y coma.

En un marco de secuentes tetralaterales, en cambio, las reglas de derivación para FDE tienen la siguiente forma general:

$$
\frac{\Gamma, A ; \Sigma, B \vdash \Delta, C ; \Theta, D}{\Xi, F ; \Pi, G \vdash \Psi, H ; \Phi, I}
$$

En el caso particular de las reglas de la negación para la lógica FDE, tenemos las siguientes cuatro reglas de derivación: 


$$
\begin{array}{ll}
\sim \mathrm{L} 1 \frac{\Gamma ; \Sigma \vdash \Delta ; \Theta, A}{\Gamma, \sim A ; \Sigma \vdash \Delta ; \Theta} & \sim \mathrm{R} 1 \frac{\Gamma, A ; \Sigma \vdash \Delta ; \Theta}{\Gamma ; \Sigma \vdash \Delta ; \Theta, \sim A} \\
\sim \mathrm{L} 2 \frac{\Gamma ; \Sigma \vdash \Delta, A ; \Theta}{\Gamma ; \Sigma, \sim A \vdash \Delta ; \Theta} & \sim \mathrm{R} 2 \frac{\Gamma ; \Sigma, A \vdash \Delta ; \Theta}{\Gamma ; \Sigma \vdash \Delta, \sim A ; \Theta}
\end{array}
$$

Si permitiéramos que las fórmulas que están en los extremos de la parte superior en las reglas $\sim \mathrm{Ll}$ y $\sim \mathrm{R} 1$ se pasaran al interior, es decir,

$$
\frac{\Gamma, A ; \Sigma \vdash \Delta ; \Theta}{\Gamma ; \Sigma, A \vdash \Delta ; \Theta} \quad \frac{\Gamma ; \Sigma \vdash \Delta ; \Theta, A}{\Gamma ; \Sigma \vdash \Delta, A ; \Theta}
$$

obtendríamos la negación de la lógica $\mathbf{K}_{3}$.

Si permitiéramos que las fórmulas que están en el interior de la parte superior en las reglas $\sim \mathrm{L} 2$ y $\sim \mathrm{R} 2$ se pasaran a los extremos, es decir,

$$
\frac{\Gamma ; \Sigma, A \vdash \Delta ; \Theta}{\Gamma, A ; \Sigma \vdash \Delta ; \Theta} \quad \frac{\Gamma ; \Sigma \vdash \Delta, A ; \Theta}{\Gamma ; \Sigma \vdash \Delta ; \Theta, A}
$$

obtendríamos la negación de la lógica LP. ${ }^{10} \mathrm{Si}$ permitiéramos las cuatro reglas de intercambio para las cuatro reglas de la negación, obtendríamos la negación clásica.

De este modo, Shapiro logra dar una caracterización de la negación independiente de otras conectivas utilizando dos marcos lógicos distintos. Cabe mencionar que los sistemas multilaterales tienen aplicaciones filosóficas bien conocidas, por ejemplo, en el contexto de lógicas no transitivas usadas para estudiar paradojas semánticas. ${ }^{11}$

La moraleja de considerar todas estas estructuras complejas es que hay una conectiva de negación cuyas propiedades están caracterizadas por la lógica misma, independientemente de cualquier otra conectiva, sólo que esto no se observa en un marco como el de conclusiones múltiples bilateral, que es el que Beall presupone en su argumento.

Una posible objeción es que los sistemas de reglas con $n$ lados no son legítimos, sino que son una manera de reescribir tablas de verdad con $n$ valores. ${ }^{12}$ El cargo de ilegimitidad es recurrente contra las

${ }^{10}$ En Ripley 2013 y Barrio, Pailós y Szmuc 2018 hay presentaciones trilaterales de $\mathbf{L P}$ que usan precisamente estas reglas.

${ }^{11}$ Véanse de nuevo Ripley 2013 y Barrio, Pailós y Szmuc 2018.

${ }^{12}$ Agradecemos a un dictaminador anónimo la sugerencia de discutir esta objeción. 
generalizaciones ${ }^{13}$ y tienden a desaparecer conforme avanza el uso de la nueva maquinaria. Pero podríamos adelantar algunas razones para disipar algunas de esas dudas acerca de los sistemas multilaterales. Primero, es útil señalar que lo que se presenta como objeción tiene algo de verdad: los sistemas de reglas multilaterales pueden considerarse como una presentación alternativa de ciertas tablas de verdad; lo mismo es cierto de los sistemas bilaterales. ${ }^{14}$ Pero de ahí no se sigue que los sistemas de reglas no sean legítimos. Las interpretaciones de los cálculos usuales en términos bilaterales - donde un secuente $\Gamma \vdash \Delta$ se interpreta "no es posible afirmar todo lo que está en $\Gamma$ y rechazar algo de lo que está en $\Delta$ "— ${ }^{15}$ simplemente se extienden para contemplar otros actos de habla. Por ejemplo, Hjortland (2014) argumenta que el lado adicional en el cálculo trilateral para $\mathbf{K}_{3}$ es para el acto de abstención (ni afirmar ni negar). Como es de esperar, el acto correspondiente para el lado adicional en LP, y así completar los cuatro lados de FDE, es difícil de conceptualizar, pero Paoli (2019) ya ha esbozado algunas ideas de cómo conceptualizar el acto de afirmar y negar a la vez. Así, hay buenas razones para no desechar como ilegítimos los cálculos multilaterales; hay argumentos para decir que son por lo menos tan legítimos, si bien menos familiares, que los cálculos bilaterales.

Sin embargo, en última instancia no es necesario considerar los sistemas multilaterales para responder a Beall, ya que hay otros cálculos de secuentes para FDE, como el de Font (1997) con reglas para la negación que tampoco involucran otras conectivas:

$$
\begin{gathered}
\sim \sim \mathrm{L} \frac{\Gamma, A \vdash B}{\Gamma, \sim \sim A \vdash B} \\
\sim \frac{A \vdash B}{\sim B \vdash \sim A}
\end{gathered}
$$

Finalmente, tanto la propuesta de Shapiro como el cálculo de Font sirven para bloquear otra línea de defensa para Beall o para alguien que piense como él. En el tema del significado de las conectivas, y siguiendo algunas ideas de Gentzen, ${ }^{16}$ es una posición estándar con-

${ }^{13}$ Véanse, por ejemplo, la afirmación de Quine de que las lógicas multivaluadas suelen ser "álgebra abstracta” (1970, p. 70), no lógica, así como las críticas de Dummett contra la lógica de conclusiones múltiples (1991, p. 187).

${ }^{14}$ Véase Barceló 2008.

${ }^{15}$ Véase Restall 2015.

${ }^{16}$ Gentzen dice que "las [reglas] de introducción de una conectiva representen, por decirlo así, las definiciones de [las conectivas] en cuestión” (1964, p. 295), dejándole a las reglas de eliminación el estatus de simples "consecuencias de esas definiciones". 
siderar que las reglas de introducción de una conectiva determinen el significado de una conectiva. Una versión más estricta sería que dichas reglas fueran puras, esto es, que sólo se introdujera una conectiva. Si, como también es usual, se considera que las reglas en que las conectivas están en los secuentes superiores representan reglas de eliminación de dichas conectivas y aquellas en que las conectivas están en los secuentes inferiores representan reglas de introducción, entonces las reglas que Beall considera para la negación no determinarían el significado de conectiva alguna. Sin embargo, en los sistemas de Shapiro y de Font sí hay tales reglas de introducción puras para la negación, de modo que sí hay reglas que determinen su significado, por lo menos según la tradición gentzeniana recién esbozada.

De esta manera, aunque las objeciones a los sistemas de secuentes multilaterales fueran correctas, el cálculo de Font es un sistema en el que la negación se presenta con las características que Beall espera. Así, la conclusión de Beall acerca de la inexistencia de la negación es apresurada, y parece haber llegado a ella simplemente por no haber considerado suficientes cálculos de secuentes para FDE.

\section{Las condiciones de evaluación para la negación}

Beall no dice por qué cree que Bl es verdadera, aunque supone que es verdadera por razones lógicas. Sin embargo, su argumento nos parece más bien una reducción al absurdo de la premisa $\mathrm{Bl}$, pues según la premisa B7, la negación lógica no es exhaustiva ni excluyente. Además, el paso de B7 a B8 no es inmediato, sino que requiere un argumento como el siguiente:

B7. La negación lógica no es excluyente ni exhaustiva.

B7a. Si la negación lógica no es excluyente ni exhaustiva, es la negación de la lógica FDE.

B7b. Sin embargo no hay conectiva que de acuerdo con FDE tenga un comportamiento independiente de cualquier otra conectiva y que esté caracterizada por las siguientes reglas de secuentes de la lógica clásica:

$$
\sim \mathrm{L} \frac{\Gamma, A \vdash \Delta}{\Gamma \vdash \sim A, \Delta} \quad \sim \mathrm{R} \frac{\Gamma \vdash A, \Delta}{\Gamma, \sim A \vdash \Delta}
$$

las cuales expresan, conjuntamente, exhaustividad y exclusividad. 8. Por lo tanto, no hay negación lógica. 
La premisa $\mathrm{B} 7 \mathrm{~b}$ expresa prácticamente lo mismo que la premisa B1. No obstante, B7b no es una razón suficiente para la conclusión. Las reglas de derivación en un marco de secuentes bilateral de conclusiones múltiples — que Beall asume en su argumento - tienen la siguiente forma general:

$$
\frac{\Gamma, A \vdash B, \Delta}{\Theta, C \vdash D, \Sigma}
$$

Una regla puede leerse modeloteóricamente siguiendo estas instrucciones: ${ }^{17}$

(LADOS) Si las fórmulas están a la derecha de $\vdash$, las fórmulas son falsas y si las fórmulas están a la izquierda de $\vdash$, las fórmulas son verdaderas.

(POLOS) Todos los secuentes en la parte superior se interpretan como en (LADOS), poniendo en conjunción las fórmulas de cada secuente; cada conjunción de fórmulas se pone en disyunción con las conjunciones de fórmulas resultantes de interpretar los demás secuentes superiores como en (LADOS). La disyunción resultante de la parte superior es el antecedente de un condicional cuyo consecuente es el secuente inferior, poniendo en disyunción sus fórmulas interpretándolas como en (LADOS). ${ }^{18}$

(CONVERTIBILIDAD) (POLOS) es convertible para el caso de la negación: la instrucción de (POLOS) para el secuente superior puede ser la instrucción del secuente inferior y viceversa. ${ }^{19}$

${ }^{17}$ La lectura de las reglas no necesita tener en cuenta $\operatorname{los}$ contextos $\Gamma, \Delta$, etc.; basta considerar el lugar de las fórmulas en las reglas.

${ }^{18}$ Por ejemplo, siguiendo la instrucción de (POLOS), la regla

$$
\rightarrow L \frac{\Gamma \vdash A, \Delta \quad \Gamma, B \vdash \Delta}{\Gamma, A \rightarrow B \vdash \Delta}
$$

tiene la siguiente lectura: si el antecedente es falso o el consecuente es verdadero, el condicional es verdadero.

${ }^{19}$ La validez y el alcance de (CONVERTIBILIDAD) es muy sensible a la presentación; para una misma lógica $\mathbf{L}$ puede haber cálculos y reglas para ciertas conectivas que sí la satisfagan y otros que no. Por ejemplo, sea $\mathbf{L}$ la lógica clásica de orden cero. Su presentación estándar en cálculo de secuentes incluye las siguientes reglas de introducción de la conjunción a la izquierda (de $\vdash)$ :

$$
\wedge \mathrm{L} \frac{\Gamma, A \vdash \Delta}{\Gamma, A \wedge B \vdash \Delta} \quad \wedge \mathrm{L} \frac{\Gamma, A \vdash \Delta}{\Gamma, A \wedge B \vdash \Delta}
$$


Sabemos que, aunque estándar, esta manera de leer las reglas no es única ni está exenta de controversias. ${ }^{20}$ Por ejemplo, las reglas también pueden leerse en términos de afirmación y rechazo, cambiando sólo la lectura de (LADOS) de la siguiente manera:

(LADOS) Si las fórmulas están a la derecha de $\vdash$, las fórmulas son rechazadas, y si las fórmulas están a la izquierda de $\vdash$, las fórmulas son afirmadas. Sin embargo, ignorar esas lecturas alternativas no perjudica en nada nuestro argumento contra Beall. ${ }^{21}$

Entonces las reglas $\sim R$ y $\sim \mathrm{L}$ expresan no exhaustividad y exlusividad sino, respectivamente, lo siguiente:

$A$ es falsa si y sólo si $\sim A$ es verdadera

$A$ es verdadera si y sólo si $\sim A$ es falsa

Pero FDE tiene exactamente estas condiciones de evaluación para la negación en la semántica relacional. Sea $\mathcal{L}$ un lenguaje con un conjunto de variables proposicionales $P R O P=\left\{p_{1}, \ldots, p_{n}\right\}$ y un conjunto de conectivas $\{\sim, \wedge, \vee\}$. Una evaluación para $P R O P$ es una relación, denotada por " $\rho$ ", entre PROP y el conjunto de valores $\{1,0\}$, de tal modo que $p \rho l$ indica que $p$ se relaciona con 1 ( $p$ se relaciona con verdadero) y $p \rho 0$, que $p$ se relaciona con 0 ( $p$ se relaciona con falso). Sea $F O R M=\{A, B, C \ldots\}$ el conjunto de fórmulas de $\mathcal{L}$ definidas de la manera usual. La relación $\rho$ se extiende a FORM y al conjunto de valores $\{1,0\}$ también de la manera usual; en particular, las condiciones de interpretación para $\sim A$ en esta semántica para FDE son las siguientes:

$\sim A \rho 1$ si y sólo si $A \rho 0$

$\sim A \rho 0$ si y sólo si $A \rho 1$

Estas reglas, también conocidas como "reglas aditivas" o "reglas extensionales", no satisfacen (CONVERTIBILIDAD). Sin embargo, la versión Ketonen de introducción de la conjunción a la izquierda - ahora más conocida como "regla multiplicativa" o "regla intensional" y que en la lógica clásica es equivalente a las dos reglas anteriores-

$$
\wedge \mathrm{L}^{\prime} \frac{\Gamma, A, B \vdash \Delta}{\Gamma, A \wedge B \vdash \Delta}
$$

sí satisface (CONVERTIBILIDAD).

${ }^{20}$ Véase Paoli 2002, pp. 30-35.

${ }^{21}$ Para una discusión más detallada de cómo leer las reglas, véanse Paoli 2002, capítulo 1, sección 3, Barceló 2008, Restall 2013, Ripley 2013, Francez 2019 y Teijeiro 2020. 
que son las que obtuvimos a partir de las reglas. Que de estas condiciones no se obtengan exclusividad y exhaustividad se debe a que la relación de interpretación no es una función e incluye tanto el caso en el que $A \rho 1$ y $A \rho 0$ como el caso en el que ni $A \rho 1$ ni $A \rho 0$.

Alguien podría preguntarse si esto no es sino un mero artefacto de la semántica relacional bivaluada. La respuesta es que no. En la otra semántica habitual para FDE, que es veritativo-funcional pero tetravaluada, la tabla de verdad para la negación es la siguiente:

\begin{tabular}{c|c}
$A$ & $\sim A$ \\
\hline$\{1\}$ & $\{0\}$ \\
$\{1,0\}$ & $\{1,0\}$ \\
$\varnothing$ & $\varnothing$ \\
$\{0\}$ & $\{1\}$
\end{tabular}

que satisface exactamente las mismas condiciones de evaluación que la semántica relacional bivaluada, a saber:

$1 \in v(\sim A)$ si y sólo si $0 \in v(A)$

$0 \in v(\sim A)$ si y sólo si $l \in v(A)$

El hecho de que la semántica bivaluada relacional y la tetravaluada funcional impliquen las mismas condiciones de verdad y falsedad para la negación — sólo hay que tomar " $\rho$ " y " $\in$ " como intercambiables - usualmente queda oculto por la elección de la notación $T$ (o $t$ ) para $\{1\}, F$ (o $f$ ) para $\{0\}, N$ (o $n$, por "neither true nor false") para $\varnothing$ у $B$ (o $b$, por "both true and false") para $\{1,0\} .{ }^{22}$

No obstante, es preciso mencionar que, siguiendo las instrucciones que adoptamos para leer modeloteóricamente las reglas de derivación, las reglas para la negación en el cálculo de Font no inducen estas condiciones, sino, respectivamente, las siguientes:

$A$ es verdadera si y sólo si $\sim A$ es verdadera

$A$ es falsa si y sólo si $\sim \sim A$ es falsa

$A$ es verdadera y $B$ es falsa si y sólo si $\sim A$ es falsa y $\sim B$ es verdadera.

Estas condiciones de evaluación para la negación no corresponden exactamente con las condiciones expresadas por las reglas $\sim \mathrm{R} y$ $\sim \mathrm{L}$ porque en la última de las tres condiciones se consideran dos fórmulas para expresar la condición de evaluación estándar de la

${ }^{22}$ Véanse Priest 2008, p. 143 y Omori y Wansing 2017 para más detalles de las semánticas para FDE. 
negación, no sólo una. Sin embargo, el cálculo de Font aventaja a los otros cálculos que hemos considerado hasta ahora en que no sólo tiene reglas puras de introducción para la negación, sino que las reglas reflejan una variante de la propiedad esperada de la negación de intercambiar verdad y falsedad.

En otras palabras: Beall afirmó que no hay una negación lógica por no encontrar reglas puras para la negación, sin siquiera sopesar que una conectiva lógica también puede ser distinguida por sus condiciones de verdad y de falsedad, y que la negación tiene condiciones de evaluación muy particulares que la distinguen de otras conectivas; pero de ser cierto que una conectiva lógica se caracteriza por reglas puras (de introducción), el cálculo de Font no sólo cumple con esta característica, sino que también las condiciones de evaluación de la negación en el cálculo de Font tienen virtualmente las mismas condiciones de evaluación que la negación en la lógica clásica. Así, encontramos más razones fuertes para afirmar que sí hay negación lógica.

Así, el argumento de Beall para concluir que no hay negación lógica no es sólido, pues no es verdad que la lógica no le imponga ninguna restricción (interesante) a la conectiva de negación más allá de lo que la lógica le demanda de su interacción con otras conectivas. Como ha quedado claro por las condiciones de evaluación para la negación, la negación tiene que intercambiar el valor de verdad por el de falsedad y viceversa, y esto es independiente de la interacción que tiene con otras conectivas.

\section{El separatismo}

En la sección 3, al discutir algunos pasos faltantes entre B7 y B8, notamos que Beall exigía que las reglas para la negación no incluyeran otras conectivas. La insistencia de Beall en que una conectiva lógica debe estar caracterizada por reglas en las que no aparezcan otras conectivas, las denominadas "reglas puras", se asemeja a la postura separatista en el tema del significado de las conectivas lógicas, y ofrece otra opción para rechazar la solidez de su argumento. Aunque la falta de solidez del argumento de Beall ya quedó establecida y no nos pronunciaremos acerca de la verdad de este supuesto adicional, creemos importante discutirlo por dos razones. La primera es que de este modo redondearíamos nuestro análisis del argumento de Beall. La segunda es que ese supuesto está estrechamente vinculado con discusiones en filosofía de la lógica que hemos abordado en este 
artículo, como el tema del significado de las conectivas lógicas, y el de la negación en particular.

De acuerdo con el separatismo, el significado de cada conectiva lógica se determina de manera independiente del significado de otras conectivas. El separatismo se opone al interaccionismo, postura según la cual el significado de cada conectiva lógica depende de las relaciones que tenga con otras conectivas. Aunque es similar a la distinción entre minimalismo y maximalismo semánticos, la distinción entre separatismo e interaccionismo es independiente. El maximalismo es la tesis según la cual el significado de una conectiva $c$ en una lógica $\mathbf{L}$ bajo una presentación $P$ depende de todos los elementos de $\mathbf{L}$ (bajo $P)$ que estén relacionados con $c$; el minimalismo es la tesis según la cual el significado de $c$ no depende de todos esos elementos. La presentación $P$ suele ser alguna versión de una teoría de pruebas, siguiendo el trabajo de Paoli (2003), aunque también hay versiones modelo teóricas como en Beall y Restall 2006 y Estrada González $2011 .^{23}$ Si $P$ es, digamos, un cálculo de secuentes tipo Gentzen para $\mathbf{L}$, un maximalista diría que tanto las reglas estructurales para $\mathbf{L}$ como las operacionales para $c-\mathrm{y}$ quizá todos los secuentes derivables en los que aparezca $c$ - determinan al significado de ésta, mientras que un minimalista diría que sólo las reglas operacionales lo hacen.

El separatismo no es necesariamente minimalista. Para el separatismo, el resto de conectivas en $\mathbf{L}$ no contribuye a determinar el significado de $c$, pero está abierta la posibilidad de que las reglas estructurales sí lo hagan. Y el interaccionismo no es necesariamente maximalista. El ejemplo de interaccionismo que Routley tiene en mente al introducir esa noción es el de las lógicas conexivas. Los esquemas distintivos de esas lógicas son las tesis de Aristóteles $\sim \sim(A \rightarrow \sim A)$ $\mathrm{y} \sim(\sim A \rightarrow A)-\mathrm{y}$ de Boecio $-(A \rightarrow B) \rightarrow \sim(A \rightarrow \sim B) \mathrm{y}$ $(A \rightarrow \sim B) \rightarrow \sim(A \rightarrow B)$ - Según Routley, en esas lógicas los significados de la negación y del condicional son inseparables, no se puede dar el significado de la una sin dar también el del otro y viceversa. Pero eso no implica maximalismo: no es necesario que el significado de la negación esté determinado por el de otras conectivas más allá del condicional y tampoco por las reglas estructurales, si es que las lógicas conexivas se presentaran en un cálculo de Gentzen. ${ }^{24}$

Cada una de estas posturas puede ejemplificarse tanto en teoría de pruebas como en teoría de modelos. En el interaccionismo en

${ }^{23}$ Para una discusión más reciente del debate maximalismo-minimalismo, véanse Hjortland 2014 y Estrada González 2020.

${ }^{24}$ Para una discusión más extensa del separatismo y el interaccionismo, véanse Routley et al. 1982 (p. 93) y Estrada González 2020. 
teoría de pruebas, el significado de las conectivas se determina por las reglas (básicas, no derivables ni admisibles) y axiomas en las que aparecen, pero en esas reglas y axiomas puede aparecer más de una conectiva. Los sistemas axiomatizados al estilo Hilbert son tal vez el caso paradigmático de interaccionismo, porque si el significado de las conectivas está determinado por los (esquemas de) axiomas en los que aparecen, ese significado típicamente estaría determinado por el de otras conectivas. Pero el interaccionismo no es sugerido sólo por los sistemas axiomáticos tipo Hilbert. En el cálculo de secuentes que Beall usa para presentar FDE, una de las reglas básicas para la negación es la siguiente:

$$
\sim \wedge \mathrm{R} \frac{\Gamma \vdash \Delta, \sim A, \sim B}{\Gamma \vdash \Delta, \sim(A \wedge B)}
$$

Tal regla es claramente interaccionista: si el significado de la negación estuviera determinado por esa regla, dependería del significado de la conjunción. $^{25}$

El separatismo en teoría de pruebas es más común. Por ejemplo, en sistemas de secuentes de Gentzen y en sistemas de deducción natural es habitual encontrar reglas en las que sólo aparece una sola conectiva. También es común en teoría de modelos. Por ejemplo, las condiciones de evaluación de cada conectiva casi siempre son dadas en términos independientes de otras conectivas, como en la lógica FDE. No obstante, también hay teorías de modelos interaccionistas, como la semántica bivaluada de Suszko para la lógica trivaluada de Łukasiewicz $^{26}$ en la que encontramos, por ejemplo, las siguientes condiciones de evaluación para el condicional, en las que también aparece la negación:

Si $v(A)=v(B)$ y $v(\sim A)=v(\sim B)$ entonces $v(A \rightarrow B)=1$ Si $v(A)=v(B)$ y $v(\sim A) \neq v(\sim B)$ entonces $v(A \rightarrow B)=v(\sim A)^{27}$

Como ya dijimos, Beall presupone una perspectiva similar al separatismo en teoría de pruebas, pero no tanto acerca del significado de las conectivas sino en cuanto a su logicidad. Según ese tipo de separatismo, una conectiva es lógica si y sólo si las reglas básicas en

${ }^{25}$ Aunque Beall no trata explícitamente el tema del significado de las conectivas, que las reglas para la negación tengan esta característica es parte de lo que lleva a Beall a sostener que no hay negación lógica.

${ }^{26}$ Véase Suszko 1975.

${ }^{27}$ Otro ejemplo de esto son las semánticas bivaluadas que desarrollan da Costa y Alves (1977) para las lógicas $\mathbf{C}_{n}$. 
las que aparece no contienen otras conectivas. Beall no ofrece ninguna razón para defender este tipo de separatismo. Como vimos, en lugar de concluir la inexistencia de la negación lógica, pudo o bien haber considerado una presentación alternativa de FDE o haber cuestionado su concepción separatista de la logicidad de una conectiva.

\section{Conclusiones}

En este artículo discutimos dos razones para dudar de la primera premisa en el argumento de Beall. La primera razón es que hay reglas para la negación en las que no aparecen otras conectivas en marcos de secuentes diferentes a los considerados por Beall, pero estas reglas no expresan exclusividad y exhaustividad. La segunda razón dice que no es verdad que la negación tenga que ser excluyente o exhaustiva. La negación bien puede ser involutiva, es decir, intercambiar el valor de verdad por el de falsedad (y viceversa), y ésta es una restricción interesante sobre la negación.

De haber un argumento sólo en contra de la negación lógica, éste tendría que considerar todas las caracterizaciones que se han dado de la negación lógica. Beall sólo considera una de esas caracterizaciones, y por eso su argumento falla. ${ }^{28}$

\section{BIBLIOGRAFÍA}

Barceló, A., 2008, "Patrones inferenciales", Crítica, vol. 40, no. 140, pp. 335.

Barrio, E., F. Pailós y D. Szmuc, 2018, “A Recovery Operator for Nontransitive Approaches", Review of Symbolic Logic, vol. 13, no. 1, pp. 80-104.

Beall, Jc., 2018, "The Simple Argument for Subclassical Logic", Philosophical Issues, vol. 28, no. 1, pp. 30-54.

, 2017, "There Is No Logical Negation: True, False, Both and Neither", Australasian Journal of Logic, vol. 14, no. 1, pp. 1-29. -, 2009, Spandrels of Truth, Oxford University Press, Oxford.

${ }^{28}$ Este trabajo se realizó en el marco del proyecto PAPIIT IN403719 "Intensionalidad hasta el final: un nuevo plan para la relevancia lógica". Presentamos versiones previas en el Logic Colloquium 2019 y en el Seminario de Tesistas en Filosofía de las Ciencias Formales (FiCiForTes), a cuyas audiencias agradecemos por sus comentarios y sugerencias. El primer autor también recibió el apoyo de una beca del Consejo Nacional de Ciencia y Tecnología (Conacyt) para estudios de posgrado. El primer autor también agradece al Seminario de Tesistas en Aprietos por sus comentarios y sugerencias. Finalmente, agradecemos los muy útiles comentarios de dos dictaminadores de la revista porque nos ayudaron a mejorar sustancialmente el artículo. 
Beall, Jc y G. Restall, 2006, Logical Pluralism, Oxford University Press, Oxford.

Berto, F., 2015, “A Modality Called 'Negation”", Mind, vol. 124, no. 495, pp. 761-793.

Berto, F. y G. Restall, 2018, "Negation on the Australian Plan", Journal of Philosophical Logic, vol. 48, no. 6, pp. 1119-1144.

da Costa, N.C.A. y E.H. Alves, 1977, "A Semantical Analysis of the Calculi Cn", Notre Dame Journal of Formal Logic, vol. 18, no. 4, pp. 621-630.

De, M. y H. Omori, 2018, "There Is More to Negation Than Modality", Journal of Philosophical Logic, vol. 47, pp. 281-299.

Dummett, M., 1991, The Logical Basis of Metaphysics, Harvard University Press, Cambridge, Mass.

Estrada González, L., 2020, "The Bochum Plan and the Foundations of Contra-Classical Logics", CLE e-Prints, vol. 19, no. 1, pp. 1-22.

- 2011, "On the Meaning of Connectives: Apropos of a NonNecessitarianist Challenge", Logica Universalis, vol. 5, no. 1, pp. 115126.

Field, H., 2008, Saving Truth from Paradox, Oxford University Press, Oxford.

Font, J.M., 1997, "Belnap's Four-Valued Logic and De Morgan Lattices", Logic Journal of the IGPL, vol. 5, no. 3, pp. 413-440.

Francez, N., 2019, "Bilateralism, Trilateralism, Multilateralism and Polysequents", Journal of Philosophical Logic, vol. 48, pp. 245-262.

Gentzen, G., 1964, "Investigations into Logical Deduction", American Philosophical Quarterly, vol. 1, no. 4, pp. 288-306.

Hjortland, O., 2014, "Speech Acts, Categoricity, and the Meanings of Logical Connectives", Notre Dame Journal of Formal Logic, vol. 55, no. 4, pp. 445-467.

Kremer, M., 1988, "Kripke and the Logic of Truth", Journal of Philosophical Logic, vol. 17, no. 3, pp. 225-278.

Marcos, J., 2005, “On Negation: Pure Local Rules", Journal of Applied Logic, vol. 11, pp. 185-219.

McGee, V., 1991, Truth, Vagueness and Paradox: An Essay on the Logic of Truth, Hackett, Indianápolis.

Omori, H. y H. Wansing, 2017, “40 Years of FDE: An Introductory Overview", en H. Omori y H. Wansing (eds.), Studia Logica, vol. 105, pp. 1021-1049.

- (eds.), 2019, New Essays on Belnap-Dunn Logic, Springer, Berlín.

Paoli, F., 2019, "Bilattice Logics and Demi-Negation", en H. Wansing y H. Omori (eds.), New Essays on Belnap-Dunn Logic, Springer, Berlín, pp. 233-253.

—_ 2003, "Quine and Slater on Paraconsistency and Deviance", Journal of Philosophical Logic, vol. 32, pp. 531-548.

, 2002, Substructural Logics: A Primer, Kluwer, Dordrecht. 
Priest, G., 2008, An Introduction to Non-Classical Logics, Cambridge University Press, Cambridge.

— 2006, In Contradiction: A Study of the Transconsistent, 2a. ed., Oxford University Press, Oxford.

—_, 1979, "The Logic of Paradox", Journal of Philosophical Logic, vol. 8, no. 1, pp. 219-241.

Quine, W.V.O., 1970, Philosophy of Logic, Prentice Hall, Englewood Cliffs, Nueva Jersey.

Restall, G., 2015, "Assertion, Denial, Accepting, Rejecting, Symmetry and Paradox", en C. Caret y O. Hjortland (eds.), Foundations of Logical Consequence, Oxford University Press, Oxford, pp. 310-321.

—_, 2013, "Assertion, Denial and Non-Classical Theories", en K. Tanaka y col (eds.), Paraconsistency: Logic and Applications. Logic, Epistemology, and the Unity of Science, Springer, Dordrecht.

Ripley, D., 2013, "Paradoxes and Failures of Cut", Australasian Journal of Philosophy, vol. 91, no. 1, pp. 139-164.

Routley, R. y col., 1982, Relevant Logics and their Rivals, vol. 1, Ridgeview, Atascadero, Cal.

Shapiro, L., 2016a, "LP, K3, and FDE as Substructural Logics", en P. Arazim y T. Lavicka (eds.), The Logica Yearbook 2015, College Publications, Londres.

— , 2016b, "The Very Idea of a Substructural Approach to Paradox", Synthese. (doi: 10.1007/s11229-016-1230-x).

Suszko, R., 1975, "Remarks on Lukasiewicz's Three-Valued Logic", Bulletin of the Section of Logic, vol. 4, no. 3, pp. 87-90.

Teijeiro, P., 2020), "Not a Knot", Thought: A Journal of Philosophy, vol. 9, no. 1, pp. 14-24.

Weber, Z., 2010, "A Paraconsistent Model of Vagueness", Mind, vol. 119, no. 476 , pp. 1025-1045.

Weber, Z. y col., 2014, "Tolerating Gluts", Mind, vol. 123, no. 491, pp. 813-828.

Recibido el 9 de marzo de 2020; aceptado el 8 de agosto de 2020. 